\title{
Characteristics of a Magnetic Bulk Thermostat for Granular Gas Investigations in Microgravity
}

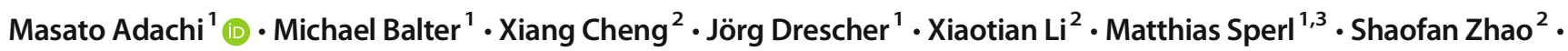 \\ Peidong $\mathrm{Yu}^{1,3}$
}

Received: 9 July 2020 / Accepted: 1 December 2020 / Published online: 18 January 2021

(C) The Author(s) 2021

\begin{abstract}
A magnetic thermostat employing soft-ferromagnetic particles and a varying magnetic field has been developed to investigate a homogeneous granular gas system in microgravity. While the thermostat's mechanism of creating homogeneous distribution of the particles was shown earlier, its characteristics have not been understood well due to limited access to a microgravity environment. Therefore, a parametric study by numerical simulation based on the discrete element method is carried out in this paper to evaluate effects of tunable parameters in the thermostat. The result shows the capability of the system and provides a wide range of options and improvements for future experiments. Moreover, it predicts that the thermostat allows variation of homogeneity and excitation level of the granular gas just by changing the magnetic parameters without using any mechanical means. In addition, the suggested improvement is experimentally implemented and evaluated in a drop tower test.
\end{abstract}

Keywords Granular gas · Granular shaking · Magnetic thermostat $\cdot$ Drop tower experiment $\cdot$ Discrete element method

\section{Introduction}

Granular gases are systems where sparsely distributed particles dissipate their energies through collisions with each other (Brilliantov and Pöschel 2004; Das et al. 2016; Goldhirsch and Zanetti 1993; Harth et al. 2018; Herrmann et al. 2001). These systems have been investigated to answer questions of granular physics, that can be found in nature on Earth and in space environments, e.g. grain motions in sandstorms and mechanisms of interstellar dust agglomerations. Insights of granular physics are also valuable for industrial optimization of granular handling processes and for In-Situ Resource Utilization (ISRU) to enable long-term space explorations (Crawford 2015; Opsomer et al. 2017; Sanders and Larson

Masato Adachi

Masato.Adachi@dlr.de

1 Institut für Materialphysik im Weltraum, Deutsches Zentrum für Luft- und Raumfahrt (DLR), 51170 Köln, Germany

2 Qian Xuesen Laboratory of Space Technology, China Academy of Space Technology, Beijing 100094, China

3 Institut für Theoretische Physik, Universität zu Köln, 50937 Köln, Germany
2013). ISRU requires mining and chemical processing of local soils on the Moon and Mars, extracting indispensable resources from the soils, such as metals, water, and oxygen, in low gravity where the particle mobility increases as that in granular gas.

Investigations of the gas state necessitates the challenging condition that particles distribute homogeneously and randomly under external excitations in microgravity to maintain the gaseous state (Brilliantov and Pöschel 2004). Thus, the investigations have been carried out thoroughly by simulations (Cafiero et al. 2000, 2002; Cafiero and Luding 2000; Kang et al. 2010; Li et al. 2011; Miller and Luding 2004; Mitrano et al. 2012; RubioLargo et al. 2016), while several experimental investigations have been performed in microgravity platforms e.g. drop tower, parabolic flight, sounding rocket, and magnetic levitation, where particles were excited by means of mechanical-boundary shaking or magnetic force (Aumaître et al. 2018; Evesque et al. 2005; Falcon et al. 1999, 2013; Harth et al. 2013; Hou et al. 2008; Maaß et al. 2008; Olafsen and Urbach 1998; Rouyer and Menon 2000; Sack et al. 2013; Wang et al. 2018; Yu et al. 2019). In addition, an analytical method for granular gas investigations was developed aided by machine learning technique to evaluate structural and dynamic properties of the particles (Puzyrev et al. 2020).

Unlike boundary shaking, magnetic excitation is a noncontact method applying force to particles in the bulk. One 
way of magnetic excitation is to employ soft ferromagnetic particles and several electromagnets (Yu et al. 2019). The electromagnets are switched on and off alternately in time sequence, and the varying magnetic field drags the particles in different directions to increase the granular excitation level as a bulk thermostat. The experiment was performed in microgravity and it achieved a reasonably homogeneous spatial distribution of particles moving in random directions ( $\mathrm{Yu}$ et al. 2019). The granular dynamics during the excitations was simulated by numerical calculation based on the discrete element method (DEM) (Cundall and Strack 1979), considering mechanical and magnetic interactions between the constituent particles (Adachi et al. 2019). The simulation reveals that the thermostat utilizes magnetic interactions between particles to create the homogeneous state. In addition, the velocity distribution during the subsequent cooling process obtained from the experiment was discussed in depth in comparison with the kinetic theory of gases (Yu et al. 2020). The thermostat does not require any mechanically moving parts, which is an advantage for applications in harsh environments, and thus ideal for future granular gas experiments in microgravity.

Although basic mechanisms of the magnetic excitation were clarified by the experiment and the simulation (Adachi et al. 2019; Yu et al. 2019), the characteristics of the thermostat have not been understood extensively, due to limited chances of experiments in microgravity. Further understanding of the bulk thermostat is required to prepare and optimize future investigations. Therefore, a parametric study using DEM simulation is conducted in this research, and it serves (1) to evaluate and explain effects of tunable key parameters in the thermostat and (2) to explore possibilities of further improvement or manipulation of the states of granular gases for future experiments. In the following sections, we shall begin with a brief review of the setup, followed by an explanation of the numerical simulation, and end with results and discussions. The investigated topics on the thermostat are categorized into the sample cell geometry, the magnetic parameters, and the granular bulk condition. In addition, one improvement suggested by the simulation is experimentally demonstrated by a drop tower test.

\section{Magnetic Bulk Thermostat}

Figure 1 shows the set-up of the magnetic bulk thermostat (Yu et al. 2019). Spherical soft-ferromagnetic particles with high relative magnetic permeability are confined in a cubic sample cell or a spherical sample cell. Four or eight commercial electromagnets are placed surrounding the sample cell (Fig. 1a, c), and they are turned-on and -off in the time sequence shown in Fig. 1b, $\mathrm{c}$ to agitate the particles in different directions. Two magnets facing each other are turned on for time $t_{E} \mathrm{~ms}$ while the others are turned off. After a resting time $t_{R} \mathrm{~ms}$ for all

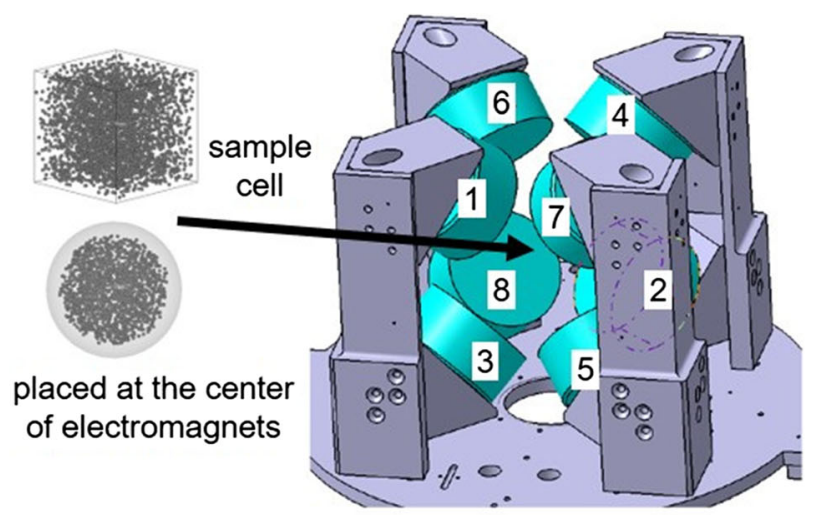

(a)

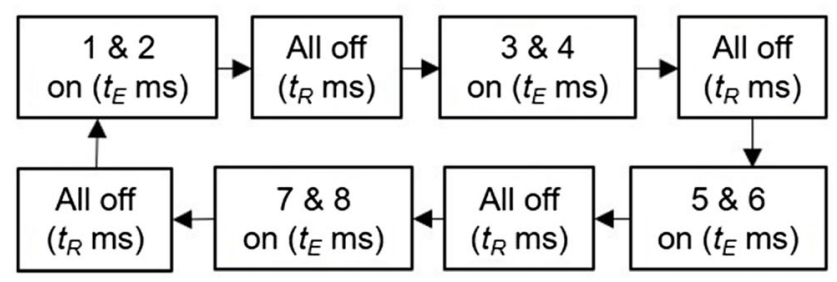

(b)
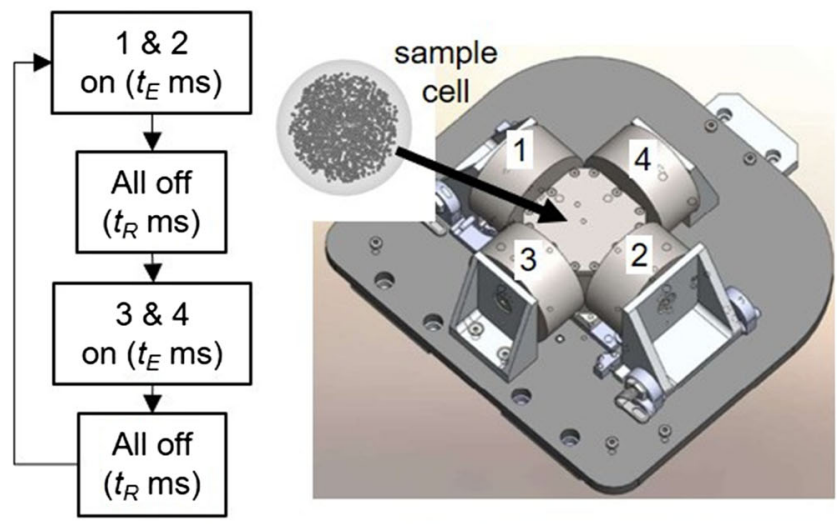

(c)

Fig. 1 Schematics of a magnetic bulk thermostat. a The setup consists of a sample cell and 8 electromagnets, and the electromagnets are turned-on and -off alternately in $\mathbf{b}$ the time sequence to elevate an excitation level of particles $\left(t_{E}\right.$ : turning-on time of one pair of electromagnets, $t_{R}$ : turning-off of all electromagnets). c 4-electromagnet set-up used for a drop tower experiment and the operation time-sequence

electromagnets, another pair of magnets is activated at the next phase. During the turning-on phase, the magnetic particles are magnetized and interact with each other via long range interactions, while during the turning-off phase, they become demagnetized and their interaction forces become negligible. A homogeneous distribution of the magnetic particles is realized by the intermittent operation using multiple electromagnet pairs due to three mechanisms: the first is the magnetic interactions between particles, which alleviates the preference of the external magnetic field-direction and broadens directions of magnetic forces applied to particles; the second is 
the switching of the electromagnet pairs for long-term isotropic shaking; the third is the random collisions between particles during the turning-off phase that absorb kinetic energies of excessively excited particles and randomize the particle motions (see the reference for details (Adachi et al. 2019)). For the simulation, the 8-magnet set-up is reproduced as standard condition, and effects of the key parameters are investigated in this paper.

\section{Numerical Calculation}

The particles during the magnetic excitations are simulated by 3D DEM based on the hard sphere contact model (Cundall and Strack 1979; Deen et al. 2007; Hoomans et al. 2001). The detailed explanation can be found in the reference (Adachi et al. 2019). The equation of motion for the $i$-th particle affected by the sum of the $j$-th force $\boldsymbol{F}_{i, j}$ can be represented as Eq. (1),

$m_{i} \ddot{\mathbf{x}_{\mathbf{i}}}=\sum_{j} \mathbf{F}_{i, j}$

where $m_{i}$ and $\boldsymbol{x}_{i}$ are the $i$-th particle mass and the positional coordinate, respectively. The velocity and position of the particle are calculated by solving Eq. (1) at each time step (1.0 $\times$ $10^{-5} \mathrm{~s}$ ) using the Runge-Kutta method. Particle-particle and particle-sample cell collisions are calculated using the modified hard sphere model (Adachi and Kawamoto 2017), based on the coefficient of restitution $\varepsilon$ set for each collision case. The particles placed initially in a sample cell are agitated by magnetic excitations. The magnetic force $\boldsymbol{F}_{i, m a g}$ applied to $i$-th particle is calculated by using Eq. (2) and it is inserted into the right-hand side of Eq. (1) (Jones 1995),

$\mathbf{F}_{i, \operatorname{mag}}=\left(\mathbf{p}_{i} \cdot \nabla\right) \mathbf{B}_{i}$

Here, $\boldsymbol{p}_{i}$ and $\boldsymbol{B}_{i}$ are the magnetic dipole moment induced in $i$-th particle, and the magnetic flux density at the particle position, respectively. Assuming one magnetic dipole moment $\boldsymbol{p}_{\boldsymbol{i}}$ in one particle, the dipole moment can be calculated by Eq. (3) (Jones 1995),

$\mathbf{p}_{i}=\frac{4 \pi}{\mu_{0}} \frac{\mu_{i}-1}{\mu_{i}+2} R_{i}^{3} \mathbf{B}_{i}$

where $\mu_{0}, \mu_{\mathrm{i}}$, and $R_{i}$, are the magnetic permeability of free space $\left(1.25 \times 10^{-6} \mathrm{H} / \mathrm{m}\right)$, the relative magnetic permeability of $i$-th particle, and the particle radius, respectively. Directions of the induced moment $\boldsymbol{p}_{\boldsymbol{i}}$ and the field $\boldsymbol{B}_{\boldsymbol{i}}$ are equal, and the magnetic force causes a translational motion on the particle. The field $\boldsymbol{B}_{\boldsymbol{i}}$ involves an external field $\boldsymbol{B}_{e x t}$ from the electromagnets and an additional field $\boldsymbol{B}_{k i}$ from the $k$-th magnetized particle (Kawamoto and Hiratsuka 2009), as represented in Eq. (4),
$\mathbf{B}_{i}=\mathbf{B}_{\text {ext }}+\sum_{k=1, i \neq k}^{N} \mathbf{B}_{k i}$

Here, $N$ is the particle number. The external magnetic field $\boldsymbol{B}_{\text {ext }}$ is calculated using the finite difference method to reproduce the experimental magnetic field. The calculation procedure involving the magnetic field $\boldsymbol{B}_{k i}$ is explained in the reference (Kawamoto and Adachi 2013; Kawamoto and Hiratsuka 2009). The calculated total magnetic field $\boldsymbol{B}_{\boldsymbol{i}}$ is applied to the particle in the same time sequence explained in the last section. Because the particle has a fast response to the magnetic field, the magnetic force is ignored when the electromagnets are turned off. In addition, the air $\operatorname{drag} \boldsymbol{F}_{a}$ and the gravitational force $\boldsymbol{F}_{g}$ are neglected because the magnetic bulk thermostat is operated in vacuum and microgravity. Table 1 shows the calculation parameters. The basic parameters in Table 1, which were tested in the previous works except for the sample cell geometry (Adachi et al. 2019; Yu et al. 2019), are constantly employed without any notice of variation in the following section.

\section{Results and Discussions}

\section{Geometric Effect on a Particle Distribution}

A cubic sample cell has been employed to confine the particles in the previous work, and the accumulation of particles at the corners of the cell has been of concern for further precise investigations (Adachi et al. 2019; Yu et al. 2019). Therefore, the geometric effect of the sample cell is investigated here. Figure 2 shows the behaviors of excited magnetic particles in cases of using the cubic cell and the spherical cell (also see Supplementary Videos 1 and 2). As shown in Fig. 2c, f, particles are randomly distributed as initial condition for same volume fraction (5\%). Figure 2a, b show the calculated average velocities of the excited particles with respect to time when the coefficient of restitution between particles is varied. The fluctuation frequency of the average velocity corresponds to the varying external magnetic field, and the excitation state is saturated soon after starting the operation in all the conditions. The average velocity increases in the cubic-cell case because particles located near the cell corners are closer to electromagnets than those in the spherical-cell case and experience stronger attractive force. In addition, Fig. 2d, g show that particles are distributed more homogeneously in the spherical-cell case at the end of the excitations, while some of them prefer staying near the corners in the cubic cell. It can be also seen clearly in Fig. 2e, h, that at the end of the excitations in each case, the particles populate more densely at the corners of the cubic cell than those in the center, while no such difference can be observed for the spherical cell. 
Table 1 Calculation parameters of 3D DEM simulation

\begin{tabular}{ll}
\hline $\begin{array}{l}\text { sample cell geometry } \\
\text { inner dimension }[\mathrm{mm}]\end{array}$ & $\begin{array}{l}\text { cubic, spherical (basic) } \\
\text { cubic: } 50 \times 50 \times 50 \\
\text { spherical: } 25 \text { (radius, basic) } \\
\text { cubic: } 3000(5 \%) \\
\text { particle number (volume fraction) }\end{array}$ \\
& $1570(5 \%$, basic), $3140(10 \%), 4710(15 \%)$ \\
& 0.8 \\
particle radius $R[\mathrm{~mm}]$ & $1.86 \times 10^{-5}$ \\
particle mass $m[\mathrm{~kg}]$ & particle-particle: $0.2,0.55$ (basic), 0.9 \\
coefficient of restitution $\varepsilon$ & particle-cell: 0.9 \\
& 10,000 \\
relative magnetic permeability of particle $\mu_{i}$ & $0.5 \mathrm{x}, 1.0 \mathrm{x}$ (same as the experiment, basic), 2.0x \\
magnetic field strength & $1,2,3,5,10,20$ (basic), 40, 60, 80,100 \\
turning-on duration of magnetic excitation $t_{E}[\mathrm{~ms}]$ & $99,98,97,95,90,80$ (basic), 60, $40,20,0$ \\
turning-off duration of magnetic excitation $t_{R}[\mathrm{~ms}]$ & 5000 \\
duration of rounds of magnetic excitations $[\mathrm{ms}]$ & random distribution (basic), sedimentation \\
initial condition of particle position &
\end{tabular}

The homogeneity of the distribution is further evaluated quantitatively. Figure 3 shows the spatial probabilities and the average velocities of particles located at different distances from the center of the sample cell. Figure $3 a, b$ show that the probabilities of the particles with all the coefficient of restitution are spatially equal in the spherical-cell case, while those become higher in the outer part of the cubic cell due to the concentration of particles near the corners. It can also be seen in Fig. 3a that particle concentration is more prominent for lower coefficient of restitution in the cubic cell. Similar trends can be seen for the average velocities plotted in Fig. 3c, d. The lower velocities at the corners of the cubic cell are mainly caused by the excessive energy-dissipating collisions due to higher concentrations there (Fig. 3c), but for the spherical cell the dissipation is well mitigated (Fig. 3d). Thus, the smoothness of the spherical-cell geometry is effective to prevent particles from getting concentrated. Figure $3 \mathrm{e}, \mathrm{f}$ show the velocity distributions in $\mathrm{x}-, \mathrm{y}-\mathrm{z}$-direction at the end of magnetic excitations with Gaussian fits in each respective case. The particles move almost omnidirectionally in both cases, and the distribution is closer to the Gaussian fit in the spherical-cell case. From the results, use of the spherical cell realizes more a homogeneous distribution of particles regardless of the coefficient of restitution. It is an advantage for granular gas investigations because the coefficient of restitution is a crucial property regarding the instability towards particle clustering (Goldhirsch and Zanetti 1993; Herrmann et al. 2001; Miller and Luding 2004). In the following sections, only the spherical cell is employed.

\section{Control of an Excitation Level of a Granular Gas}

One of the advantages on the magnetic bulk thermostat is using a magnetic field without any mechanical moving parts to agitate the particles and tailor excitation levels of the granular gas. Here, effects of the magnetic field parameters, mainly the magnetic field strength and the turning-on duration of the field, are investigated. Figure $4 \mathrm{a}-\mathrm{c}$ show the calculated average velocities of the particles with respect to time for different magnetic field strengths and different turning-on durations, and Fig. 4d shows the average velocities at the end of excitations (at $5 \mathrm{~s}$ ) in each case and the linear fits. The field strength varies from the same field condition used in the previous experiment ( $\mathrm{Yu}$ et al. 2019) to a 2 times stronger field, as well as a 0.5 times weaker field. The turning-on duration ranges from 0.001 to $0.1 \mathrm{~s}$, corresponding to turning-off duration from 0.099 to $0 \mathrm{~s}$, and the total duration is set to $0.1 \mathrm{~s}$ for all the cases. As shown in Fig. $4 \mathrm{a}-\mathrm{c}$, the saturated average velocity and the saturation time become higher and lower, respectively, as the magnetic field strength increases. In addition, the steady-state average velocity increases linearly with the turning-on duration, as shown in Fig. 4d, except for cases of insufficiently or excessively applying the magnetic field. When the turning-on duration and the field strength are $0.001 \mathrm{~s}$ and 0.5 times weaker respectively, which is the insufficient case, the average velocity is smaller than the linear fit in Fig. 4d because the granular gas does not reach the steady state, as shown in Fig. 4a. On the other hand, in the excessive case when the turning-on duration is set to $0.1 \mathrm{~s}$ in the 2.0 times stronger field, the average velocity is 


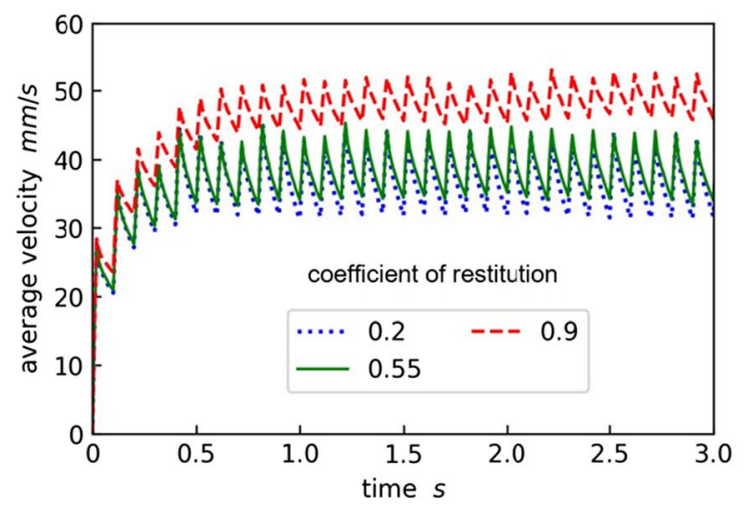

(a)

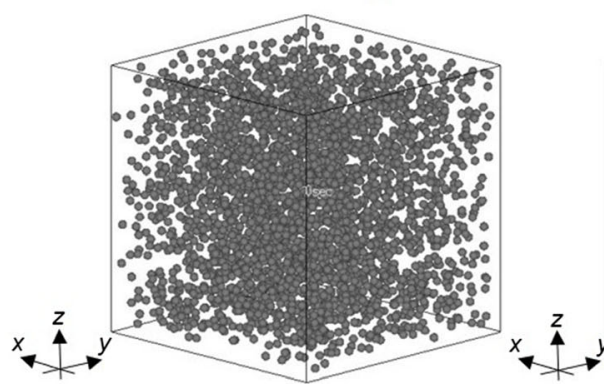

(c)

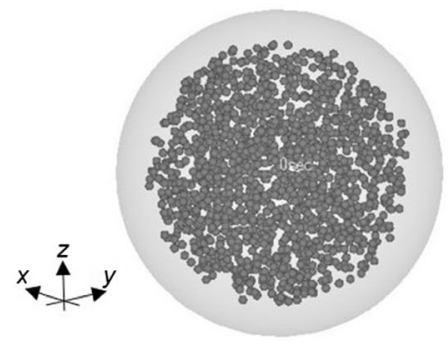

(f)

Fig. 2 Particle behaviors during magnetic excitations. a, b Average velocities of particles with respect to time during the beginning of the excitations when the coefficient of restitution between particles are varied, in cases of using $\mathbf{a}$ a cubic cell and $\mathbf{b}$ a spherical cell. Snapshots of the particles at $\mathbf{c}, \mathbf{f}$ the initial condition $(0 \mathrm{~s})$ and at $\mathbf{d}, \mathbf{g}$ the end of excitations ( $5 \mathrm{~s}$ ) in each case (The coefficient of restitution: 0.2). Spatial distributions of the particle densities at the ends of rounds of non-excited phases, which are accumulated $\mathbf{e}$ in $x$-direction in the cubic-cell case and $\mathbf{h}$

beyond the linear fit. It is because the particles interact intensively, creating their clustering due to the excessive magnetic force as shown in Fig. 4e, then moving as an aggregate (also see Supplementary Video 3). Since the aggregated particles move more similarly in terms of the directions and the velocities, energy dissipations on collisions between particles become smaller. Thus, the excessive case specifically causes the higher average velocity, as shown in Fig. $4 \mathrm{c}$, d.

Figure 5 shows the spatial probabilities and the spatial average-velocities of particles in each case at the end of excitations. When the granular gas does not reach the

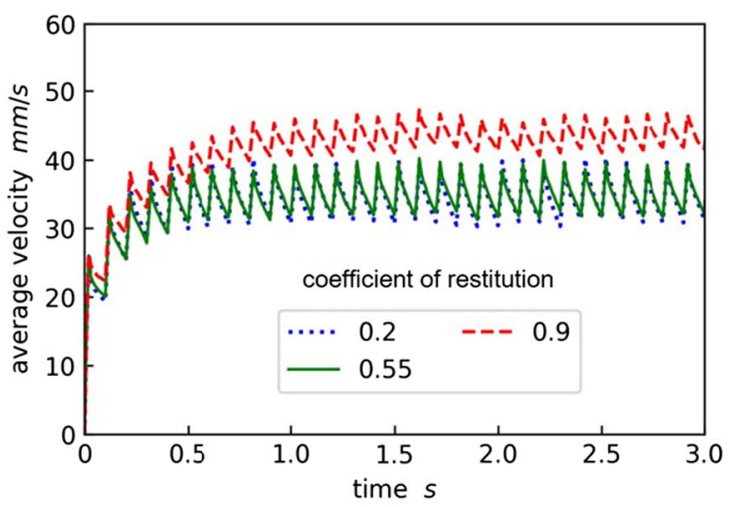

(b)

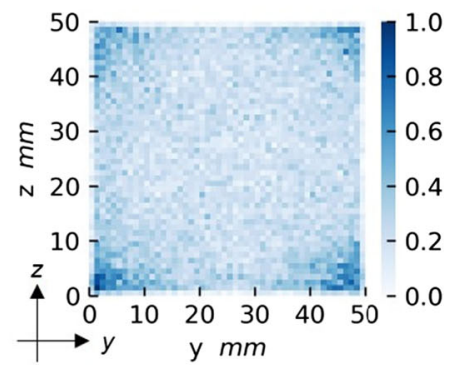

(e)

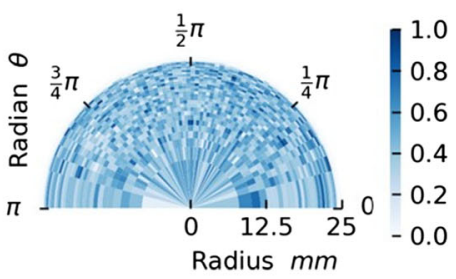

(h)

(g)

in rotational direction around $x$-axis in the spherical-cell case (The coefficient of restitution: 0.2 ). The particle densities measured at 10 different frames are superimposed for a better clarity (at $4.1 \mathrm{~s}, 4.2 \mathrm{~s}, \ldots$, $5.0 \mathrm{~s}$ ). One-time frame data contains e 3000 and $\mathbf{h} 1570$ number of particles in cubic and spherical cell cases, respectively, and the superimposed data are shown for $\mathbf{e} 30,000$ and $\mathbf{h} 15,700$ number of particles for each case

steady state (e.g. Figure 5a, turning-on duration: $0.001 \mathrm{~s}$, field strength: 0.5 times), it shows an inhomogeneous probability distribution. When the effect of the magnetic field is excessively large (e.g. Figure 5e, turning-on duration: $0.1 \mathrm{~s}$, field strength: 2.0 times), particles tend to populate highly near the sample cell boundary. The results indicate that an optimal combination of the magnetic field strength and the applied duration is crucial to create a homogeneous distribution. In addition, as long as the optimal combination is applied, the thermostat realizes tailored excitation levels of the granular gas just by adjusting the magnetic parameters. 


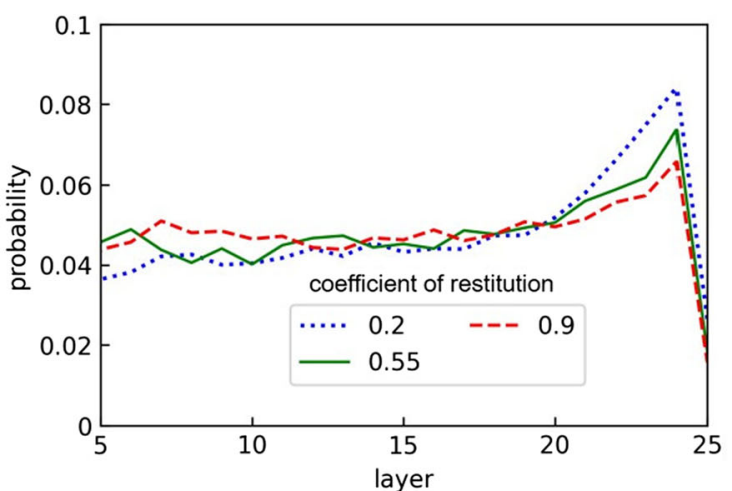

(a)

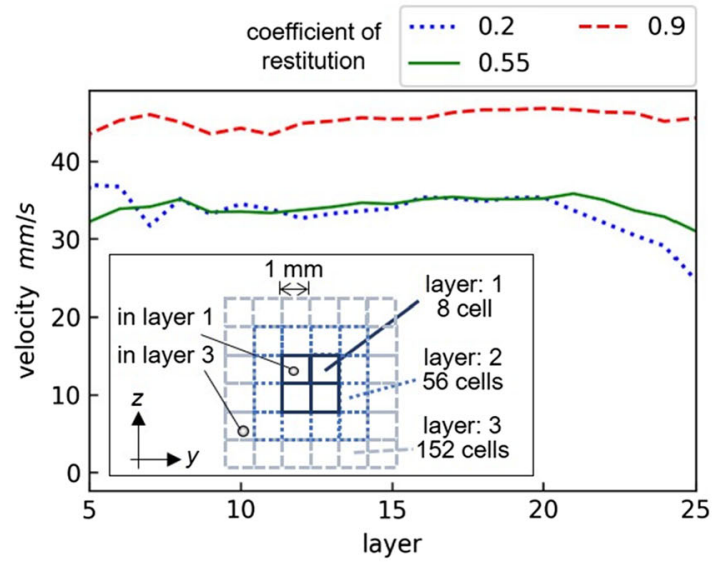

(c)

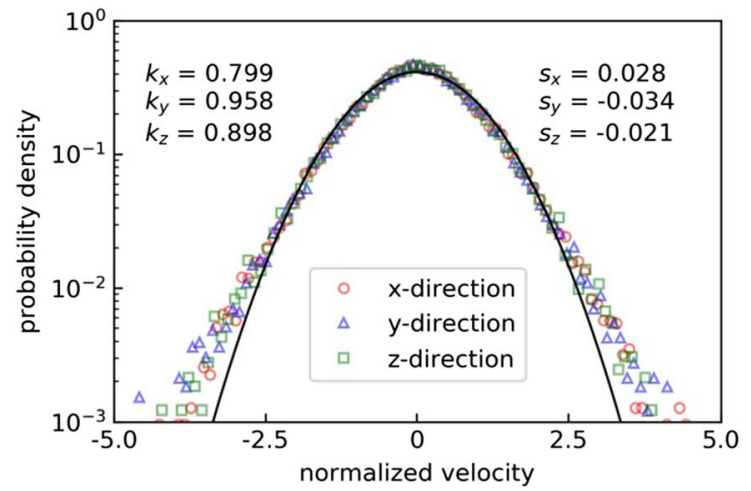

(e)

Fig. 3 Homogeneity of excited particles in cases of a cubic cell and a spherical cell. a, b Spatial probabilities and $\mathbf{c}, \mathbf{d}$ a spatial average-velocity of the particles existing in each virtual layer when the coefficient of restitution between particles is varied. The virtual layer, as shown in $\mathbf{c}, \mathbf{d}$, is used in a-d to sort particles spatially in terms of the distance from the center of the sample cell. Each layer has a $1 \mathrm{~mm}$ width, and the sample cells are filled with the

\section{Effects of Granular Bulk Condition}

The initial spatial distribution of the particles in the sample cell before the magnetic excitation cannot be known due to microgravity effects and disturbances from the platform (e.g. vibration during parabolic flights (Yu et al. 2019)). In addition, the volume fraction of the granular gas is also an

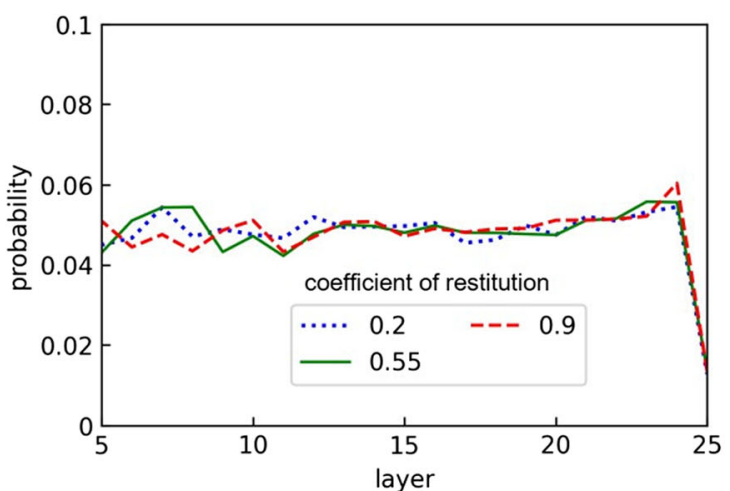

(b)

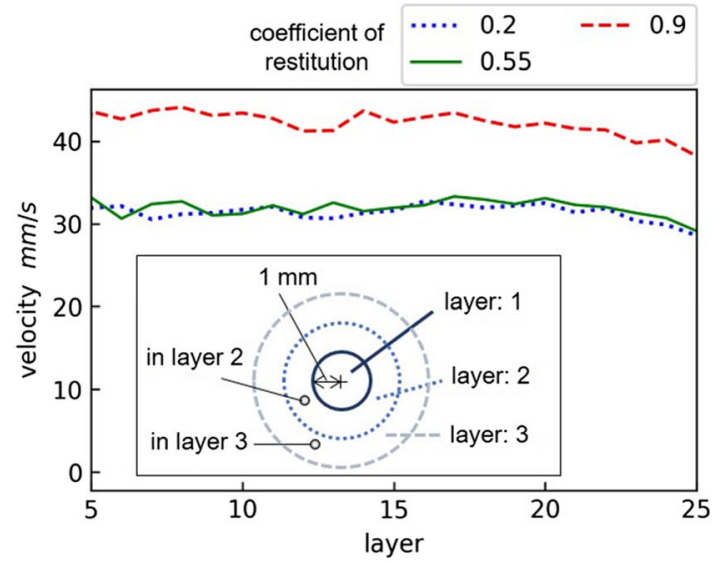

(d)

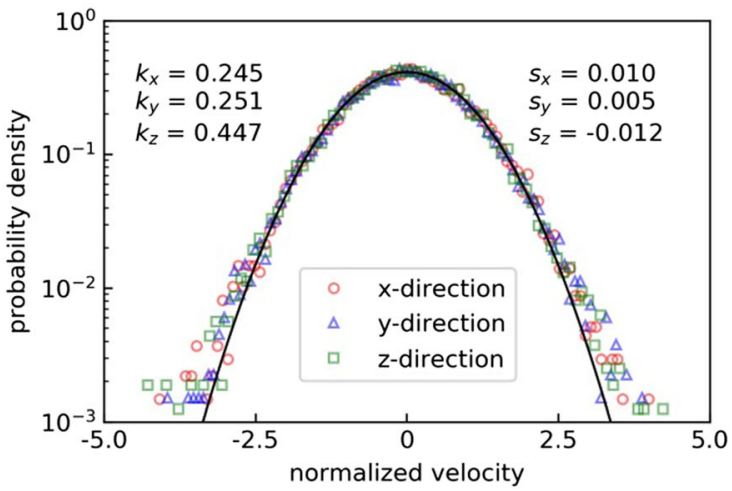

(f)

layers to leave no space within the cells. $\mathbf{e}, \mathbf{f}$ Normalized velocity probabilities in each $x$-, $y$-, $z$-direction with Gaussian fits (The coefficient of restitution: 0.2 ), and the skewness $s$ and the kurtosis $k$ (subtracted by 3 ) of the distributions. The data are obtained at the ends of rounds of non-excited phases for 10 frames and are superimposed for better statistics in all the figures (at $4.1 \mathrm{~s}, 4.2 \mathrm{~s}, \ldots, 5.0 \mathrm{~s}$ )

important parameter affecting the cooling behavior. Thus, effects of the granular bulk condition are investigated. Figure 6 shows the granular behaviors when the initial positions of the particles are altered. Two extreme initial conditions, that of an already randomly distributed gas and that of a completely sedimented packing are used. Figure 6a shows the calculated average velocities of the particles with respect to time in both 


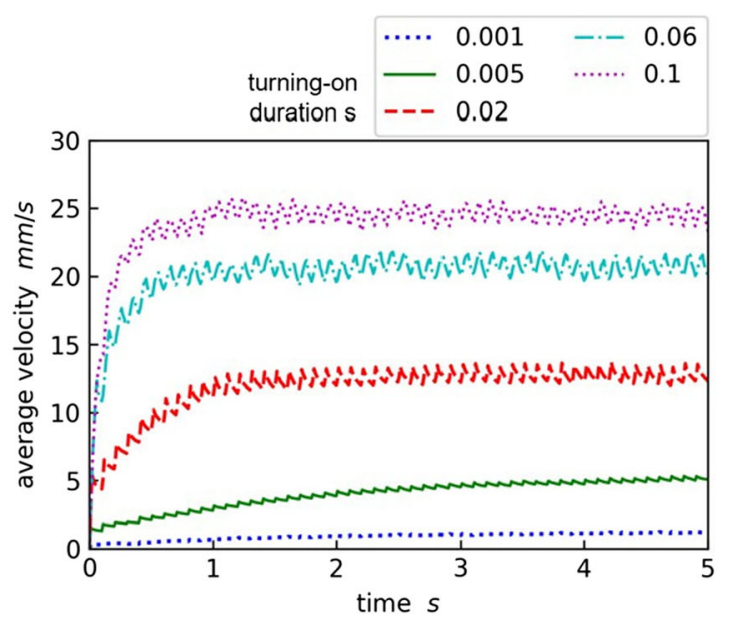

(a)

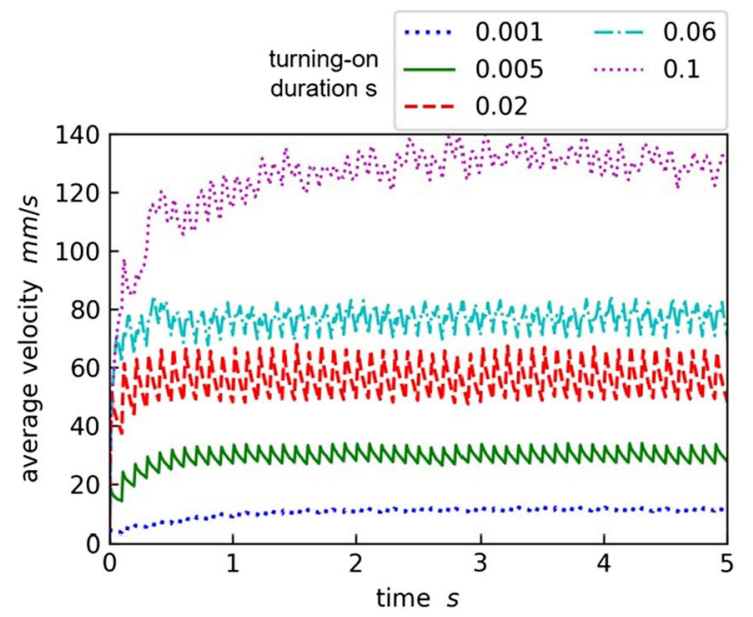

(c)

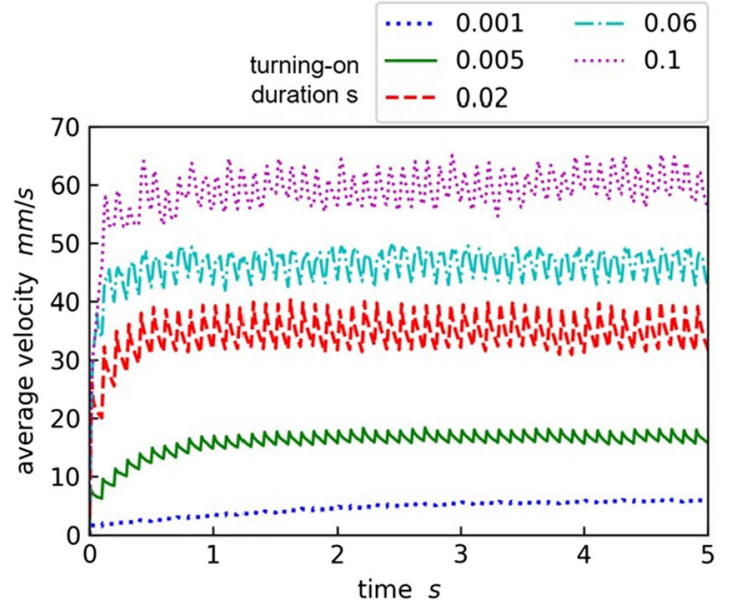

(b)

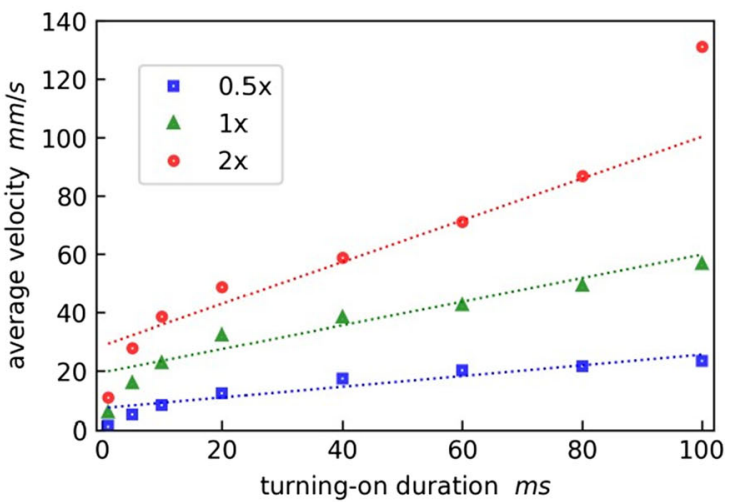

(d)

(e)

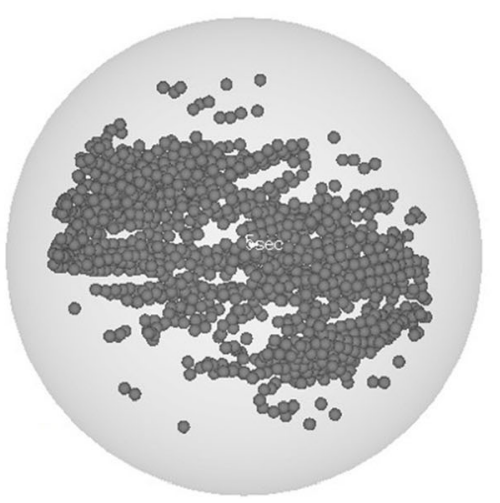

Fig. 4 Average velocities of excited particles when a magnetic-field strength and a turning-on duration of the field are varied in a sphericalcell case. The average velocities with respect to time when the turning-on duration is varied, in cases of intensifying the magnetic field to $\mathbf{a} 0.5 \mathrm{x}, \mathbf{b}$
$1.0 \mathrm{x}$, and $\mathbf{c} 2.0 \mathrm{x}$. d Average velocities of particles at the end of excitations (at $5.0 \mathrm{~s}$ ) in each case with linear fits. e Snapshots of the particles at the end of excitations (at $5.0 \mathrm{~s}$, intensification: 2.0x, turning-on: $0.1 \mathrm{~s}$ )

ending up the homogeneous distribution (also see Supplementary Video 4). Figure 6b shows the spatial probabilities of the particles, which are uniformly distributed in both cases. We may therefore conclude that wherever particles are placed as initial condition, they reach the homogeneous state within several rounds of magnetic excitations. 
Fig. 5 Homogeneity of excited particles when a magnetic-field strength and a turning-on duration of the field are varied in a spherical-cell case. a, c, e Spatial probabilities and $\mathbf{b}, \mathbf{d}, \mathbf{f}$ spatial average-velocities of the particles existing in each layer (see Fig. 3d for the explanation on the layer), in cases of intensifying the magnetic field to $\mathbf{a}, \mathbf{b} 0.5 \mathrm{x}, \mathbf{c}, \mathbf{d}$ $1.0 \mathrm{x}$, and $\mathbf{e}, \mathbf{f} 2.0 \mathrm{x}$. For better statistics, the data are obtained at the ends of rounds of non-excited phases for 10 frames (at $4.1 \mathrm{~s}$, $4.2 \mathrm{~s}, \ldots, 5.0 \mathrm{~s})$ and are superimposed
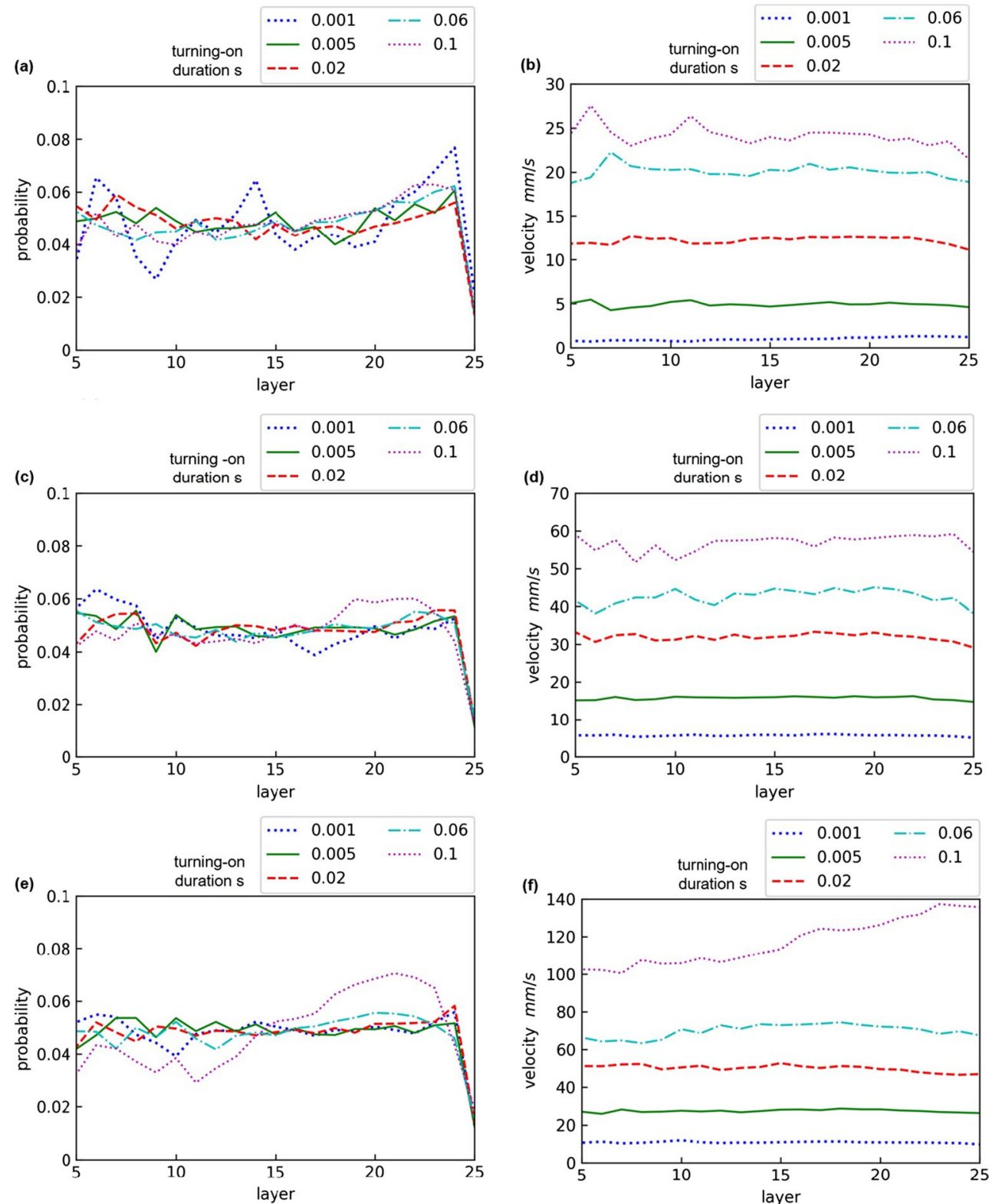

Figure $7 \mathrm{a}, \mathrm{b}$ show the spatial probabilities and the spatial average-velocities of particles when the volume fraction is varied from $5 \%$ to $15 \%$, which can be deemed granular gas state. Although the particle mobility decreases with increase of the volume fraction, the particles are distributed homogeneously even at high volume fraction, as shown in the snapshot of particles at the end of excitations (Fig. 7d). As shown in Fig. 7b, the average velocity of the particles differentiates in each case because the energies of particles dissipate substantially in case of higher volume-fraction due to more frequent collisions between particles. Figure $7 \mathrm{c}$ shows the velocitydirection distribution in volume fraction of $15 \%$. Although the average velocity is varied depending on the volume fraction, the velocity direction is randomized and omnidirectionally in all the cases.

\section{Experimental Demonstration Using a Spherical Cell}

Testing and optimizing all these parameters in experiments require a long-term low-gravity campaign, e.g. an orbital space experiment. Before that, we have the chance to use the drop tower facility operated by the Center of Applied Space Technology and Microgravity (ZARM) located in Bremen, Germany. The drop tower offers $\sim 10^{-6} \mathrm{~g}\left(1 \mathrm{~g}=9.8 \mathrm{~m} / \mathrm{s}^{2}\right)$ gravity condition for $\sim 9.4 \mathrm{~s}$. While such short time period is not enough to extensively test many different parameter sets, we use it for one demonstration: the change of sample cell geometry. As shown in Fig. 8a, the spherical drop tower test sample cell is made of polymethyl methacrylate (PMMA) and has an inner diameter of $58 \mathrm{~mm}$. About 3000 iron based amorphous alloy particles (Fe52Co20B20Si4Nb4) were placed inside. The 


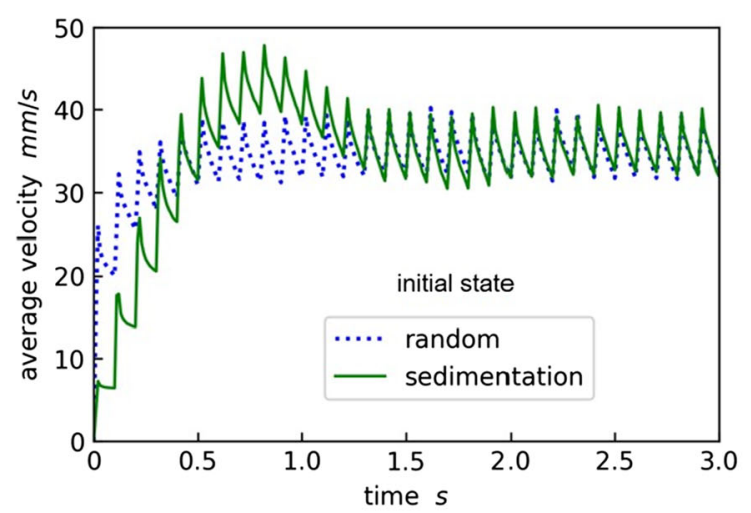

(a)

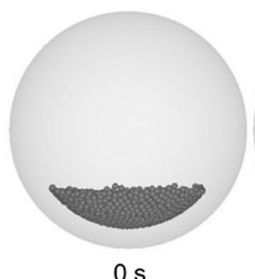

$0 \mathrm{~s}$

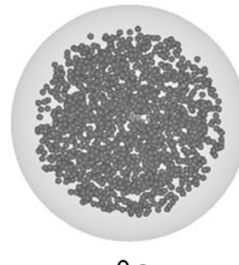

$0 \mathrm{~s}$

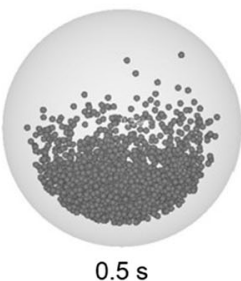

$0.5 \mathrm{~s}$
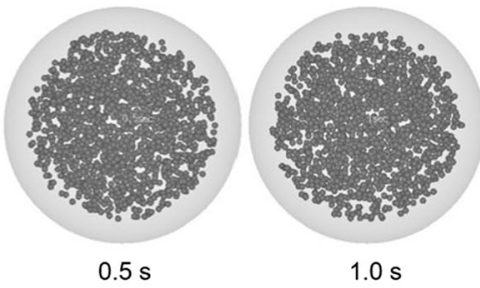

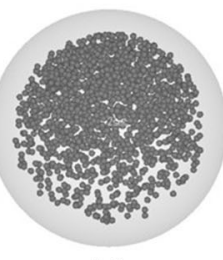

$1.0 \mathrm{~s}$

(c)
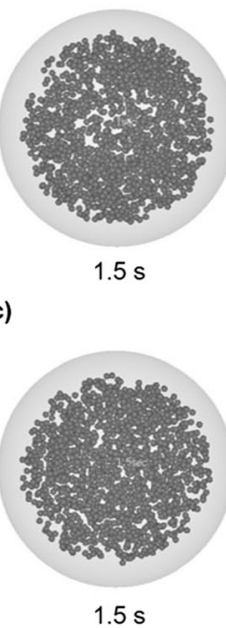

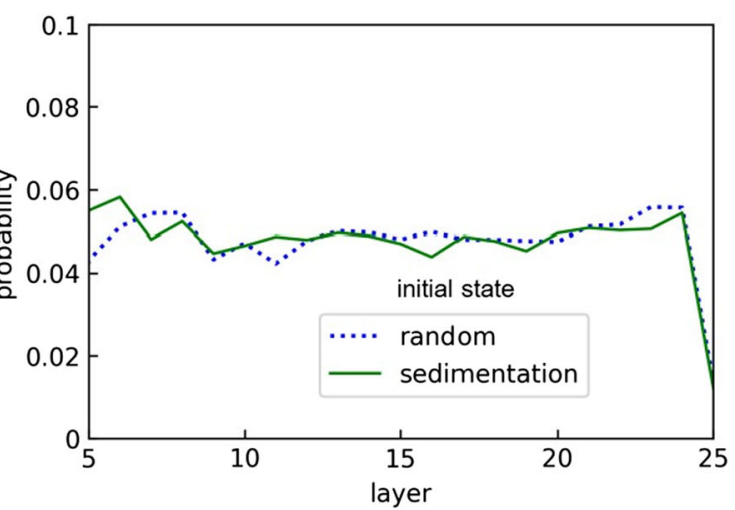

(b)
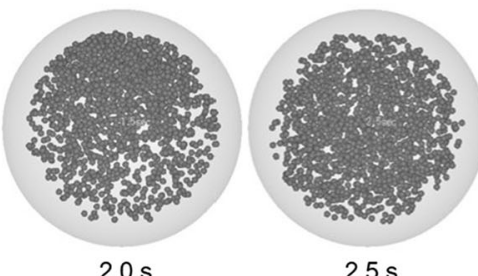

$2.5 \mathrm{~s}$
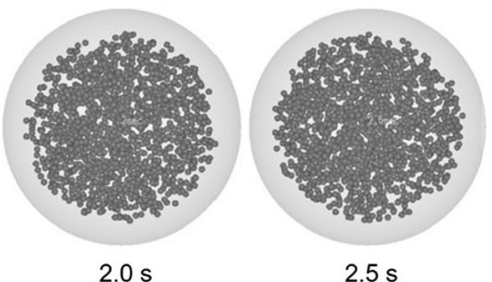

(d)

Fig. 6 Particle behaviors during magnetic excitations when an initial distribution of particle positions is varied. a Average velocities of particles with respect to time during the beginning of the excitations. $\mathbf{b}$ Spatial probabilities of the particles existing in each layer at the ends of rounds of non-excited phases (see Fig. $3 \mathrm{~d}$ for the explanation on the

particles were prepared by centrifugal granulation that leads to a spherical shape with diameter of $0.5 \mathrm{~mm}$. The amorphous alloy material has a high effective magnetic permeability and a low coercivity, which ensure the particles to quickly respond to the magnetic field without being permanently magnetized (Yu et al. 2019), thus reproduce the scenarios assumed by the simulations: particles interact with each other only under the external field but not without it. The 4-magnet setup is used for this drop tower test, as shown in Fig. 1c. Despite using only 4 magnets (turning-on: $20 \mathrm{~ms}$, turning-off: $80 \mathrm{~ms}$ ) instead of 8 in the simulation due to limited resources available for the test, within $6 \mathrm{~s}$ into the micro-g phase, the system is able to drive the particles to a nicely homogeneous distribution. The removal of corners from a cubic cell prevents any observable concentration of particle close to the 4 magnets, as better illustrated by the processed Fig. 8b (also see Supplementary Videos 5 and 6). In addition, the spatial probability of particles located at different distances from the center of the spherical cell is shown in Fig. $8 \mathrm{c}$. It also exhibits no concentration of particles near the surface layer). For better statistics, the data are obtained for 10 frames (at $4.1 \mathrm{~s}$, $4.2 \mathrm{~s}, \ldots, 5.0 \mathrm{~s}$ ) and are superimposed. Snapshots of the excited particles at each time in cases of $\mathbf{c}$ sedimentation and $\mathbf{d}$ random distribution as initial condition

of the spherical cell in the experiment, while the particles populate densely in the outer part of the cubic cell (in particular, in layers 20-24) in the simulation, as shown in Fig. 3a. Although there is a difference between experimental and simulation conditions, which needs to be eliminated for further comparison and deeper analysis of experiments in the future work, this demonstration proves that use of a spherical sample cell is promising for the preparation of granular gases distributed homogeneously.

\section{Conclusion}

A numerical simulation based on 3D DEM considering magnetic interactions between particles was carried out to understand the basic characteristics of the magnetic bulk thermostat as well as show the capability of the system for future investigations of granular gases. The calculation results show individual effects of tunable parameters of the thermostat on the 
Fig. 7 Homogeneity of excited particles when the volume fraction is varied in a sphericalcell case. a Spatial probabilities and $\mathbf{b}$ spatial average-velocities of the particles existing in each layer (see Fig. 3d for the explanation on the layer). c Normalized velocity probabilities in each $x$-, $y$-, $z$-direction with Gaussian fits (volume fraction: 15\%), and the skewness $s$ and the kurtosis $k$ (subtracted by 3 ) of the distributions. The data are obtained at the ends of rounds of non-excited phases for 10 frames and are superimposed for better statistics (at $4.1 \mathrm{~s}, 4.2 \mathrm{~s}, \ldots, 5.0 \mathrm{~s}$ ). d Snapshots of the particles at the end of excitations (at $5.0 \mathrm{~s}$, volume fraction: $15 \%$ )

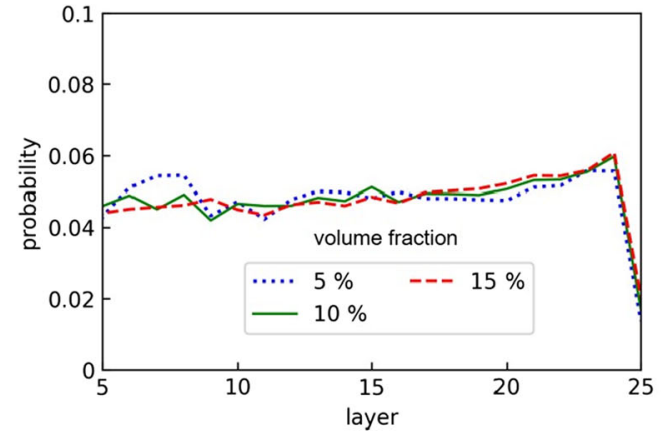

(a)

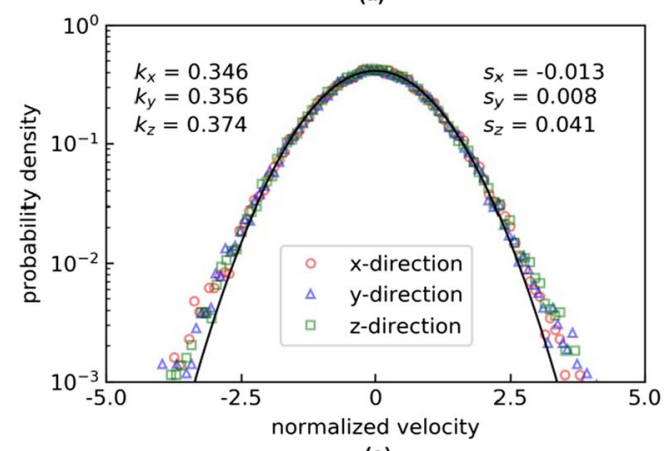

(c)

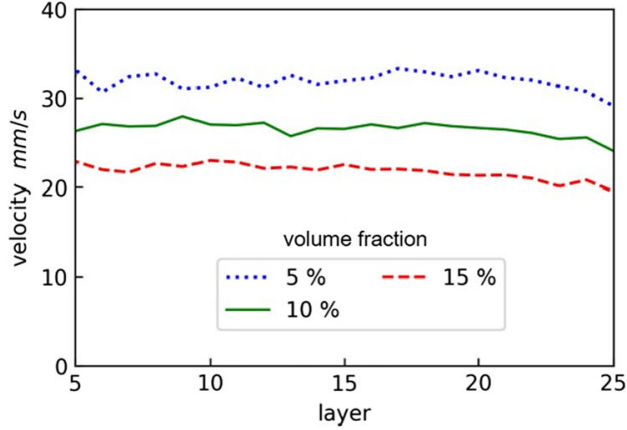

(b)

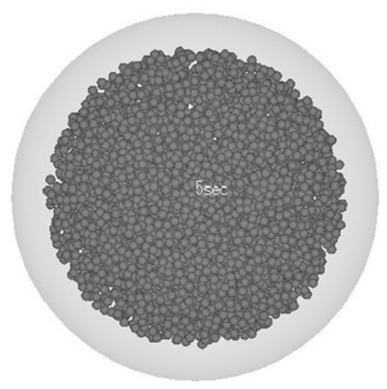

(d)
Fig. 8 Particle distribution in a spherical sample cell from a drop and " 4 " mark the positions of 4 magnets used for excitation. $\mathbf{b}$ Particle positions after image processing. c A spatial probability of the particles existing in each virtual layer. As opposed to simulation cases, each layer has a $1.16 \mathrm{~mm}$ width in analysis of the experimental result and sorts particles spatially in terms of the distance from the center of the spherical cell. Since the experimental video provides particle motions in a limited range of depth, this analyzed data shows 2D information. Light scattering and reflection on the cell surface prevent capturing motions of particles in layers closest to the boundary (layers 24, 25 that are painted with light-blue color in $\mathbf{b}$, c) are neglected. The data of particle positions are superimposed for 130 frames, and 97,010 detected particle positions are analyzed in total for better statistics tower test. a The original image, where the numbers " 1 ", "2", " 3 " particles near the boundary, and

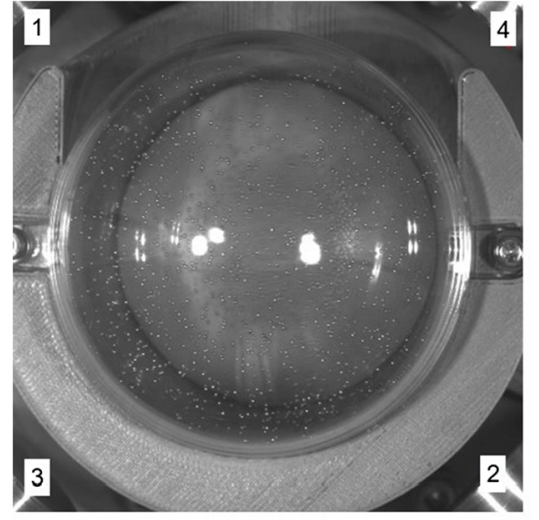

(a)

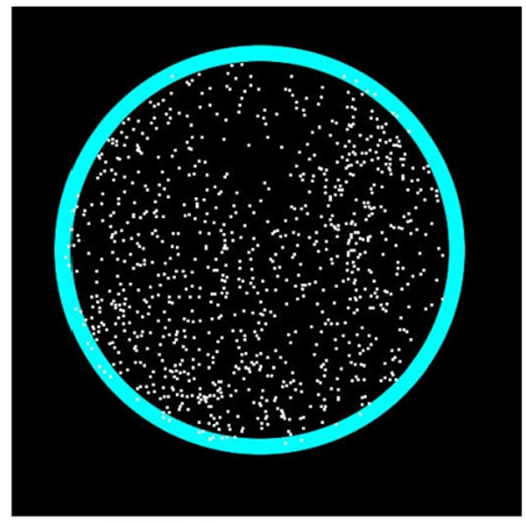

(b)

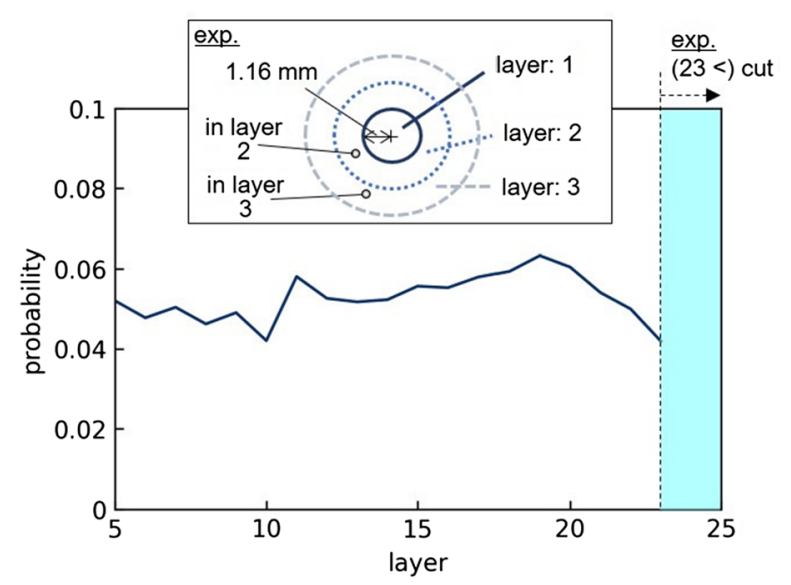

(c) 
homogeneity and the excitation level of the granular gas. From the results, changing the sample-cell geometry to spherical shape is promising for improvement of the homogeneity, and it was also demonstrated by the experiment in microgravity. Although more sophistication is necessary on the set-up, we can conclude that the use of the spherical cell facilitates more homogeneous granular gas. In addition, the simulation predicts that control of the granular gas state by adjusting magnetic parameters is possible over a wide range of parameters. In particular, the optimal combination of the magnetic field strength and the applied duration is the key to tailor excitation levels of the granular gas in homogeneous state. If longer microgravity is accommodated, various experiments can be conducted without changing the set-up or using mechanically moving parts in one campaign. The simulation also indicates that the thermostat has potential to adapt to various granular bulk conditions, such as different initial distributions and volume fractions, resulting in the creation of a homogeneous distribution of particles excited randomly and omnidirectionally.

Supplementary Information The online version contains supplementary material available at https://doi.org/10.1007/s12217-020-09853-5.

Acknowledgments We appreciate proof-reading and suggestions from Philip Born. This work is partially supported by BMWi/DLR (No. 50WM1651, 50WM1945), National Natural Science Foundation of China (No. 51901244, 51905530), and China Postdoctoral Science Foundation (No. 2018 M641440). In addition, Masato Adachi is supported by JSPS Overseas Research Fellowships and Alexander von Humboldt Research Fellowship.

Funding Information Open Access funding enabled and organized by Projekt DEAL.

\section{Compliance with Ethical Standards}

Conflict of Interest The authors declare that they have no conflict of interest.

Open Access This article is licensed under a Creative Commons Attribution 4.0 International License, which permits use, sharing, adaptation, distribution and reproduction in any medium or format, as long as you give appropriate credit to the original author(s) and the source, provide a link to the Creative Commons licence, and indicate if changes were made. The images or other third party material in this article are included in the article's Creative Commons licence, unless indicated otherwise in a credit line to the material. If material is not included in the article's Creative Commons licence and your intended use is not permitted by statutory regulation or exceeds the permitted use, you will need to obtain permission directly from the copyright holder. To view a copy of this licence, visit http://creativecommons.org/licenses/by/4.0/.

\section{References}

Adachi, M., Kawamoto, H.: Electrostatic sampler for large regolith particles on asteroids. J. Aerosp. Eng. 30, 04016098 (2017). https://doi. org/10.1061/(ASCE)AS.1943-5525.0000701
Adachi, M., Yu, P., Sperl, M.: Magnetic excitation of a granular gas as a bulk thermostat. npj Microgravity. 5, 19 (2019). https://doi.org/10. 1038/s41526-019-0079-y

Aumaître, S., Behringer, R.P., Cazaubiel, A., Clément, E., Crassous, J., Durian, D.J., Falcon, E., Fauve, S., Fischer, D., Garcimartín, A., Garrabos, Y., Hou, M., Jia, X., Lecoutre, C., Luding, S., Maza, D., Noirhomme, M., Opsomer, E., Palencia, F., Pöschel, T., Schockmel, J., Sperl, M., Stannarius, R., Vandewalle, N., Yu, P.: An instrument for studying granular media in low-gravity environment. Rev. Sci. Instrum. 89, 075103 (2018). https://doi.org/10.1063/1.5034061

Brilliantov, N.V., Pöschel, T.: Kinetic theory of granular gases. Oxford University Press. (2004)

Cafiero, R., Luding, S.: Mean field theory for a driven granular gas of frictional particles. Phys. A Stat. Mech. Appl. 280, 142-147 (2000). https://doi.org/10.1016/S0378-4371(99)00630-5

Cafiero, R., Luding, S., Jürgen Herrmann, H.: Two-dimensional granular gas of inelastic spheres with multiplicative driving. Phys. Rev. Lett. 84, 6014-6017 (2000). https://doi.org/10.1103/PhysRevLett.84. 6014

Cafiero, R., Luding, S., Herrmann, H.J.: Rotationally driven gas of inelastic rough spheres. Europhys. Lett. 60, 854-860 (2002). https:// doi.org/10.1209/ep1/i2002-00295-7

Crawford, I.A.: Lunar resources. Prog. Phys. Geogr. Earth Environ. 39, 137-167 (2015). https://doi.org/10.1177/0309133314567585

Cundall, P.A., Strack, O.D.L.: A discrete numerical model for granular assemblies. Géotechnique. 29, 47-65 (1979). https://doi.org/10. 1680/geot.1979.29.1.47

Das, P., Puri, S., Schwartz, M.: Clustering and velocity distributions in granular gases cooling by solid friction. Phys. Rev. E. 94, 032907 (2016). https://doi.org/10.1103/PhysRevE.94.032907

Deen, N.G., Van Sint Annaland, M., Van der Hoef, M.A., Kuipers, J.A.M.: Review of discrete particle modeling of fluidized beds. Chem. Eng. Sci. 62, 28-44 (2007). https://doi.org/10.1016/j.ces. 2006.08.014

Evesque, P., Palencia, F., Lecoutre-Chabot, C., Beysens, D., Garrabos, Y.: Granular gas in weightlessness: the limit case of very low densities of non interacting spheres. Microgravity Sci. Technol. 16, 280-284 (2005). https://doi.org/10.1007/BF02945991

Falcon, É., Wunenburger, R., Évesque, P., Fauve, S., Chabot, C., Garrabos, Y., Beysens, D.: Cluster formation in a granular medium fluidized by vibrations in low gravity. Phys. Rev. Lett. 83, 440-443 (1999). https://doi.org/10.1103/PhysRevLett.83.440

Falcon, E., Bacri, J.-C., Laroche, C.: Equation of state of a granular gas homogeneously driven by particle rotations. EPL Europhysics Lett. 103, 64004 (2013). https://doi.org/10.1209/0295-5075/103/64004

Goldhirsch, I., Zanetti, G.: Clustering instability in dissipative gases. Phys. Rev. Lett. 70, 1619-1622 (1993). https://doi.org/10.1103/ PhysRevLett.70.1619

Harth, K., Kornek, U., Trittel, T., Strachauer, U., Höme, S., Will, K., Stannarius, R.: Granular gases of rod-shaped grains in microgravity. Phys. Rev. Lett. 110, 144102 (2013). https://doi.org/10.1103/ PhysRevLett.110.144102

Harth, K., Trittel, T., Wegner, S., Stannarius, R.: Free cooling of a granular gas of rodlike particles in microgravity. Phys. Rev. Lett. 120, 214301 (2018). https://doi.org/10.1103/PhysRevLett.120.214301

Herrmann, H.J., Luding, S., Cafiero, R.: Dynamics of granular systems. Phys. A Stat. Mech. Appl. 295, 93-100 (2001). https://doi.org/10. 1016/S0378-4371(01)00059-0

Hoomans, B.P., Kuipers, J.A., Mohd Salleh, M., Stein, M., Seville, J.P.: Experimental validation of granular dynamics simulations of gasfluidised beds with homogenous in-flow conditions using positron emission particle tracking. Powder Technol. 116, 166-177 (2001). https://doi.org/10.1016/S0032-5910(00)00391-0

Hou, M., Liu, R., Zhai, G., Sun, Z., Lu, K., Garrabos, Y., Evesque, P.: Velocity distribution of vibration-driven granular gas in Knudsen 
Regime in microgravity. Microgravity Sci. Technol. 20, 73-80 (2008). https://doi.org/10.1007/s12217-008-9040-5

Jones, T.B.: Electromechanics of Particles. Cambridge University Press, Cambridge (1995)

Kang, W., Machta, J., Ben-Naim, E.: Granular gases under extreme driving. EPL Europhysics Lett. 91, 34002 (2010). https://doi.org/10. 1209/0295-5075/91/34002

Kawamoto, H., Adachi, M.: Numerical simulation and direct observation of dynamics of toner and carrier particles in electrophotographic two-component magnetic brush development system. J. Imaging Soc. Japan. 52, 547-554 (2013). https://doi.org/10.11370/isj.52.547

Kawamoto, H., Hiratsuka, T.: Statics and dynamics of carrier particles in two-component magnetic development system in electrophotography. J. Imaging Sci. Technol. 53, 060201 (2009). https://doi.org/10. 2352/J.ImagingSci.Technol.2009.53.6.060201

Li, Y., Hou, M., Evesque, P.: Directed clustering in driven compartmentalized granular gas systems in zero gravity. J. Phys. Conf. Ser. 327, 012034 (2011). https://doi.org/10.1088/1742-6596/327/1/012034

Maaß, C.C., Isert, N., Maret, G., Aegerter, C.M.: Experimental investigation of the freely cooling granular gas. Phys. Rev. Lett. 100, 248001 (2008). https://doi.org/10.1103/PhysRevLett.100.248001

Miller, S., Luding, S.: Cluster growth in two- and three-dimensional granular gases. Phys. Rev. E. 69, 031305 (2004). https://doi.org/ 10.1103/PhysRevE.69.031305

Mitrano, P.P., Garzó, V., Hilger, A.M., Ewasko, C.J., Hrenya, C.M.: Assessing a hydrodynamic description for instabilities in highly dissipative, freely cooling granular gases. Phys. Rev. E. 85, 041303 (2012). https://doi.org/10.1103/PhysRevE.85.041303

Olafsen, J.S., Urbach, J.S.: Clustering, order, and collapse in a driven granular monolayer. Phys. Rev. Lett. 81, 4369-4372 (1998). https://doi.org/10.1103/PhysRevLett.81.4369

Opsomer, E., Noirhomme, M., Vandewalle, N., Falcon, E., Merminod, S.: Segregation and pattern formation in dilute granular media under microgravity conditions. npj Microgravity. 3, 1 (2017). https://doi. org/10.1038/s41526-016-0009-1
Puzyrev, D., Harth, K., Trittel, T., Stannarius, R.: Machine learning for 3D particle tracking in granular gases. Microgravity Sci. Technol. 32, 897-906 (2020). https://doi.org/10.1007/s12217-020-09800-4

Rouyer, F., Menon, N.: Velocity fluctuations in a homogeneous 2D granular gas in steady state. Phys. Rev. Lett. 85, 3676-3679 (2000). https://doi.org/10.1103/PhysRevLett.85.3676

Rubio-Largo, S.M., Alonso-Marroquin, F., Weinhart, T., Luding, S., Hidalgo, R.C.: Homogeneous cooling state of frictionless rod particles. Phys. A Stat. Mech. Appl. 443, 477-485 (2016). https://doi. org/10.1016/j.physa.2015.09.046

Sack, A., Heckel, M., Kollmer, J.E., Zimber, F., Pöschel, T.: Energy dissipation in driven granular matter in the absence of gravity. Phys. Rev. Lett. 111, 018001 (2013). https://doi.org/10.1103/ PhysRevLett.111.018001

Sanders, G.B., Larson, W.E.: Progress made in lunar in situ resource utilization under NASA's exploration technology and development program. J. Aerosp. Eng. 26, 5-17 (2013). https://doi.org/10.1061/ (ASCE)AS.1943-5525.0000208

Wang, W.-G., Hou, M.-Y., Chen, K., Yu, P.-D., Sperl, M.: Experimental and numerical study on energy dissipation in freely cooling granular gases under microgravity. Chinese Phys. B. 27, 084501 (2018). https://doi.org/10.1088/1674-1056/27/8/084501

Yu, P., Stärk, E., Blochberger, G., Kaplik, M., Offermann, M., Tran, D., Adachi, M., Sperl, M.: Magnetically excited granular matter in low gravity. Rev. Sci. Instrum. 90, 054501 (2019). https://doi.org/10. 1063/1.5085319

Yu, P., Schröter, M., Sperl, M.: Velocity distribution of a homogeneously cooling granular gas. Phys. Rev. Lett. 124, 208007 (2020). https:// doi.org/10.1103/PhysRevLett.124.208007

Publisher's Note Springer Nature remains neutral with regard to jurisdictional claims in published maps and institutional affiliations. 